\title{
La calidad de empleo en la UE con perspectiva de género. Una propuesta de medida por medio de un indicador sintético*
}

\author{
VERÓNICA DE ANDRÉS SAN JOSÉ \\ Universidad de Valladolid, Facultad de Ciencias Sociales y Jurídicas, Plaza de la Universidad, 1, \\ 40005 Segovia, España. E-mail: veronica.andres.san-jose@uva.es
}

\section{RESUMEN}

Los diferentes estudios del concepto de calidad de empleo han propuesto distintas dimensiones y soluciones para su medición. Una de estas propuestas de medida es el Î́ndice Europeo de Calidad del Trabajo. Este se compone de información sobre el entorno físico y social, la intensidad y calidad del tiempo de trabajo, las diferentes habilidades e influencia que tienen los trabajadores, sus perspectivas sobre el empleo y las retribuciones recibidas por su trabajo.

Se propone a partir de las componentes de dicho índice suministradas por la European Working Conditons Survey, calcular un indicador sintético de calidad de empleo por medio de la metodología $\mathrm{DP}_{2}$, así como ofrecer una estimación robusta del indicador de calidad del trabajo, analizando qué componentes tienen un mayor peso en la determinación de la calidad del trabajo y ofrecer a su vez un análisis espacial de los niveles de calidad de empleo en la UE con una perspectiva de género.

Palabras clave: Indicador sintético, Medida de Distancia P2, calidad del empleo, género.

\section{The Quality of Employment in the EU from a Gender Perspective. A Proposal for Measurement Using a Synthetic Indicator}

\begin{abstract}
Different studies into the notion of quality of employment have proposed several dimensions and various solutions for measuring it. One such measurement proposal is the European Index of Quality of Work. This index comprises information concerning the physical and social environment, the intensity and quality of work time, the different skills involved and how workers influence their jobs, their job prospects and the earnings they receive for their work.

Based on the components included in this index provided by the European Working Conditions Survey, we propose estimating a synthetic indicator of quality of employment using the $\mathrm{P}_{2}$ distance methodology. The aim is to offer a robust estimate of the quality of employment, exploring which components carry greater weight when determining quality of employment, and also providing a spatial analysis of employment quality levels from a gender perspective.

Keywords: Synthetic Indicator, $\mathrm{DP}_{2}$ distance measure, Quality of Employment, Gender.
\end{abstract}

Clasificación JEL: J81, C43

\footnotetext{
* Una versión preliminar de este artículo fue galardonado con el premio Bernardo Pena para jóvenes investigadores durante el XXXII Congreso Internacional de Economía Aplicada, ASEPELT2018, celebrado en Huelva.
}

Artículo recibido en julio de 2018 y aceptado en septiembre de 2018

Artículo disponible en versión electrónica en la página www.revista-eea.net, ref. ə-36305 


\section{INTRODUCCIÓN}

La calidad de empleo no es un concepto nuevo que haya surgido en los últimos años, sino que desde mediados de la década de los años 70 ha ocupado una posición central en las agendas políticas de los gobiernos nacionales de los diferentes países. Inicialmente con un enfoque fundamentalmente cuantitativo, es decir, centrado en la creación de puestos de trabajo y desde la década de los 90 con un enfoque también cualitativo, como consecuencia del deterioro de las condiciones laborales que estaban experimentando cada vez más un mayor número de trabajadores, así como por las transformaciones que se estaban produciendo en el mercado de trabajo como consecuencia de la flexibilización de las formas de contratación y remuneración, la introducción de nuevas formas de gestión de las relaciones laborales y la incorporación de mujeres e inmigrantes como nuevos colectivos en el mercado de trabajo. Todos estos aspectos hicieron que los gobiernos nacionales de los diferentes países de la Unión Europea (UE) no se centraran únicamente en las cifras de empleo, como habían venido haciendo hasta los años 90, sino que comenzaran a preocuparse por las condiciones laborales de los trabajadores, es decir, por las capacidades para la formación y el desarrollo profesional, la salud y la seguridad en el trabajo, la flexibilidad, la conciliación, etc. (Begega y Guillén, 2009). La mejora de todos estos aspectos que se enmarcan dentro del concepto de calidad de empleo, hace que los trabajadores se encuentren más satisfechos, lo que implicará trabajadores más productivos, lo que repercutirá positivamente sobre los beneficios empresariales. Por ello, Green en 2006 ya señalaba que el buen empleo no era otra cosa que un vehículo para que los trabajadores alcanzasen un grado de bienestar adecuado y una serie de objetivos de carácter personal mediante la concesión de ciertas capacidades como el control personal del trabajo, la colaboración y desarrollo de trabajo en equipo, etc.

El objetivo fundamental del presente trabajo es la construcción de un indicador sintético de calidad de empleo para los individuos de la Unión Europea mediante la agregación de las siete componentes que establece la Encuesta Europea sobre Condiciones de Trabajo en el informe publicado por Eurofound (2017). Con ello, se pretende analizar si las diferencias existentes por género en los diferentes países de la UE son significativas o no para explicar las desigualdades entre hombres y mujeres en materia de calidad de empleo, lo que a su vez repercute también sobre la salud y el bienestar de los mismos. Para ello, se utilizará la metodología $\mathrm{DP}_{2}$, ya que se ha comprobado que la misma presenta una serie de características que la hacen idónea para el desarrollo de indicadores sintéticos, así como permitirnos realizar un análisis no solo a nivel interespacial sino también interindividual, lo que resulta muy interesante cuando queremos realizar comparaciones entre subgrupos poblacionales. 
Por todo ello, en primer lugar se definirá el concepto de calidad de empleo y se dará una explicación acerca de las diferentes dimensiones que integrarán el indicador sintético. Posteriormente, se presentará brevemente la metodología de la distancia P2 y se analizará la estructura del indicador sintético para determinar las diferencias entre componentes así como sus principales estadísticos. A continuación, se llevará a cabo un análisis de la distribución del indicador sintético por género y países para determinar si las diferencias existentes por género son significativas o no para explicar las desigualdades existentes en materia de calidad de empleo. Por último, se expondrán las principales conclusiones obtenidas, así como las referencias bibliográficas consultadas para la elaboración del mismo.

\section{DEFINICIÓN DEL CONCEPTO DE CALIDAD DE EMPLEO}

Lo primero que deberíamos concretar es que entendemos por calidad de empleo o empleo de calidad. La Organización Internacional del Trabajo (OIT) se refiere a la calidad del empleo como trabajo decente y lo define a partir de cuatro principios: respeto a los derechos fundamentales del trabajador y a los estándares laborales internacionales; igualdad de oportunidades para todos en cuanto a empleo y remuneración; acceso a la protección social y a la seguridad social; y diálogo social bipartido y tripartido (Somavia, 2004).

No obstante, la calidad de empleo es un concepto difícil de definir, no existiendo una definición unánime sobre el mismo. Ello es debido en parte a su carácter multidimensional, vago o difícil de precisar. Por ello, existen diversos estudios que han propuesto combinar varias dimensiones del concepto de calidad de empleo como el de Begega y Guillén (2009), Clark (2005), Arranz et.al. (2016) y Somarriba et.al. (2010). Algunos proponen abordar el enfoque multidimensional de la calidad de empleo teniendo en cuenta:

- Por un lado, las características intrínsecas del trabajo, donde incluimos la remuneración percibida, la jornada laboral, el contenido del trabajo (interés, prestigio, independencia) o las competencias exigidas para el desempeño.

- Por otro lado, los aspectos relacionados con el entorno del empleo tales como las condiciones generales de trabajo, las relaciones interpersonales en el lugar de trabajo, las posibilidades de formación, las expectativas de desarrollo profesional, las coberturas y otros beneficios sociales alcanzados a través del empleo.

Este carácter multidimensional del concepto lo hacen patente organismos internacionales como por ejemplo la Comisión Europea en 2001, que plasma el carácter multidimensional del concepto de calidad de empleo a través de un conjunto de diez dimensiones relacionadas con la calidad de vida laboral: calidad intrínseca del puesto de trabajo; cualificaciones, aprendizaje continuado 
y desarrollo profesional; igualdad de género; salud y seguridad en el trabajo; flexibilidad y seguridad; inclusión y acceso al mercado de trabajo; organización del trabajo y equilibrio con la vida cotidiana; diálogo social; diversidad y no discriminación y productividad y desarrollo económico (Arranz et.al., 2016).

Por tanto, se observa que en general todos los estudios e iniciativas que han tratado de definir el concepto de calidad de empleo recogen para ello dimensiones relacionadas no solo con el propio puesto de trabajo, sino otras relacionadas con el entorno y las condiciones laborales de los trabajadores. No obstante, cada uno considera un número y tipo de dimensiones diferente para su definición, aunque parece admitida la naturaleza multidimensional del concepto.

En conclusión, ya que el concepto de calidad de empleo resulta indirectamente medible, un posible enfoque es utilizar los indicadores sociales con el objetivo de mostrar una visión de este concepto (Somarriba et.al., 2010), siendo un aspecto fundamental para la construcción de estos indicadores tener en cuenta que el concepto de calidad de empleo se encuentra sujeto a consideraciones tanto objetivas como subjetivas.

\section{COMPONENTES DEL ÍNDICE EUROPEO DE CALIDAD DEL TRABAJO}

El objetivo fundamental del presente trabajo es ofrecer una medida de la calidad del empleo de los trabajadores de la Unión Europea (UE28), para ello se utilizan las componentes del Índice Europeo de Calidad del Trabajo (JQI, Job Quality Index) creado por el Instituto Sindical Europeo de Investigación, Educación, Salud y Seguridad (ETUI) en 2008, con el objetivo de medir la calidad del trabajo en la UE. Para ello se basa en una serie de subíndices como son: los salarios; el empleo no estándar; el tiempo de trabajo; el equilibrio vidatrabajo; la formación y la representación de intereses, los cuales intentan captar las distintas dimensiones de la calidad del trabajo. El ETUI ha actualizado en 2017 este índice con los resultados de la Encuesta Europea sobre Condiciones de Trabajo (EWCS) realizada en 2015. Piasna (2018) presenta un estudio que ha recogido la evolución experimentada por el índice y sus componentes de los países de la UE28 desde 2005 hasta 2015, observándose que ha habido una reducción de la calidad del trabajo no asalariado en las últimas décadas, así como un crecimiento lento de los salarios en los años posteriores a la crisis. Este índice presenta como inconveniente que es un promedio no ponderado de las diferentes variables, las cuales recogen componentes no solo de la EWCS sino también de la Encuesta de Población Activa Europea (LFS, Labour Force Survey) y de la base de datos sobre características institucionales de los sindicatos, el marco salarial, la intervención del Estado y los pactos sociales (ICTWSS, Database on Institutional Characteristics of Trade Unions, Wage Setting, State Intervention and Social Pacts). 
En nuestro caso, vamos a construir un Indicador Sintético de Calidad de Empleo (ISCE) a partir de las componentes recogidas en la EWCS de 2015 realizada por la Fundación Europea para la Mejora de las Condiciones de Vida y de Trabajo (Eurofound).

El ISCE estará formado por las siete dimensiones recogidas en la EWCS que reflejan la naturaleza multidimensional del concepto de calidad de empleo. Estos componentes no solo influyen en el concepto de calidad de empleo sino que sabemos que tienen influencia sobre la salud y el bienestar de los trabajadores, todos tienen una gran cantidad de características en común, ya que tratan de captar como desarrollan su trabajo los distintos trabajadores y bajo qué condiciones. Asimismo, están construidos a partir de indicadores positivos y negativos sobre las características laborales y por otra parte, tienen en cuenta las características laborales desde una perspectiva objetiva, es decir, aquellas características que pueden ser observadas. Todos los componentes del índice están medidos en la encuesta en la misma escala que va de 0 a 100 con excepción del componente ganancias, ya que este hace referencia al salario o las ganancias recibidas por el trabajo realizado. Este último componente está medido en euros. Los siete componentes del índice, como se muestra en la siguiente figura, son:

Figura 1

Componentes del Índice de Calidad de Empleo

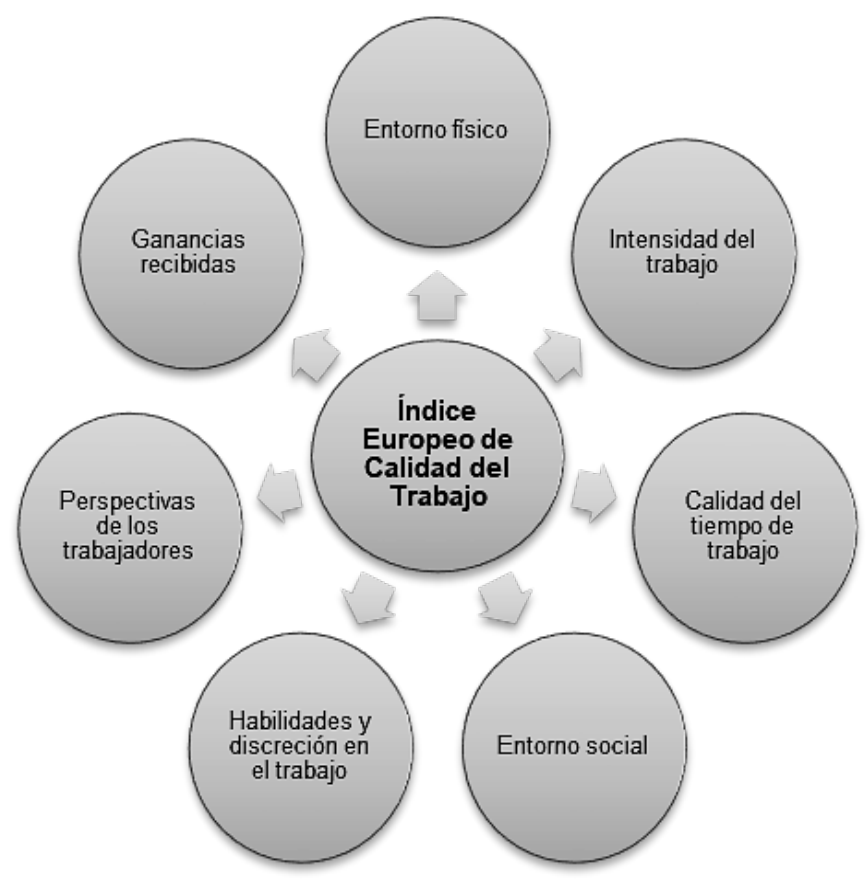

Fuente: Elaboración propia, EWCS. 
A continuación, se comentarán cada una de las diferentes componentes del índice definidas en el informe publicado por Eurofound (2017), con el fin de comprender qué aspectos mide cada una y qué diferencia arrojan desde una perspectiva de género.

\subsection{Entorno físico}

Este índice hace referencia al entorno de trabajo y a los riesgos que se pueden derivar del mismo. Se analiza en la EWCS mediante la exposición a tres tipos de riesgos: riesgos ergonómicos, relacionados con la postura que deben adoptar los trabajadores en su puesto de trabajo como son levantar personas, cargas pesadas, movimientos repetitivos...; riesgos derivados del ambiente, tales como la exposición al ruido, a las altas y bajas temperaturas, etc.; y riesgos biológicos y químicos que pueden surgir por la exposición a humos y vapores, manejo de productos químicos o materiales infecciosos, entre otros.

De los tres riesgos derivados del entorno de trabajo, el primero de ellos es el más frecuente en Europa, y además genera problemas musculo-esqueléticos en los trabajadores. El último de ellos, la exposición a riesgos químicos y biológicos se ha observado que se ha ido incrementado en los últimos años. No obstante, en términos globales si analizamos la evolución del índice en los últimos años a través de los resultados publicados en las diferentes EWCS podríamos decir que ha mejorado aunque lo ha hecho lentamente, pasando en términos globales de una puntuación media de 82 a 84 puntos.

Si analizamos el índice por género podríamos destacar que las mujeres presentan niveles más bajos de exposición a estos riesgos que los hombres, ya que normalmente estos riesgos se concentran en aquellos sectores más masculinizados.

\subsection{Intensidad del trabajo}

El componente que se refiere a la intensidad del trabajo no se mueve en la misma dirección que el resto de componentes, en el sentido de que un mayor valor implica menor calidad de trabajo, sino que tiene una asociación o impacto negativo en la calidad del empleo y por lo tanto en la salud y el bienestar de los trabajadores. Los niveles altos de intensidad del trabajo son muy comunes en Europa y la medimos a través de tres factores:

- Demandas cuantitativas de trabajo: aspectos relacionados con tener que trabajar rápido, sujeto a plazos, interrupciones, etc.

- Determinantes del ritmo e interdependencia: si únicamente el ritmo de trabajo depende del trabajador o tienen influencia otros factores como son los compañeros, los clientes, los objetivos, la maquinaria... 
- Demandas emocionales, entendiendo por estas el hecho de tener que ocultar los sentimientos en el trabajo, el tener que tratar con clientes insatisfechos o malhumorados o situaciones que puedan distorsionar emocionalmente...

Este índice, si observamos los datos publicados por Eurofound (2017) para 2015 presenta puntuaciones relativamente bajas en la mayoría de los casos, lo cual implica altos niveles de intensidad del trabajo en toda Europa. No obstante, si lo segregamos por género podemos observar que las puntuaciones obtenidas son similares en mujeres y hombres, destacando que las mujeres tienden a presentar niveles más elevados de intensidad en lo relacionado con los requerimientos de los clientes, puesto que predominan más en sectores de actividad donde el trato con el cliente es mayor. Por otra parte, los hombres tienden a experimentar niveles más elevados en lo relativo a las demandas de trabajo, ya que en las cadenas de producción, donde es fundamental el trabajo por objetivos, hay un mayor número de hombres que de mujeres. Asimismo, como ya hemos comentado estos niveles altos de intensidad influyen negativamente en la calidad del trabajo y a su vez en la salud y el bienestar de los trabajadores, ya que implican un incremento de las enfermedades cardiovasculares, musculoesqueléticas y depresiones.

\subsection{Calidad del tiempo de trabajo}

La calidad del tiempo de trabajo es un componente fundamental dentro del concepto de calidad de empleo y por tanto, desempeña un papel importante también en la salud y el bienestar de los trabajadores. Este índice se construye a través de cuatro componentes:

- La duración del trabajo: recoge cuestiones sobre si se trabajan más horas a la semana de las legalmente establecidas, si los periodos de descanso son inferiores a lo legalmente establecido, etc. Este componente tiene un impacto negativo, ya que cuanto mayor sea la duración del trabajo peor será la calidad del mismo.

- Tiempo de trabajo atípico: incluye las cuestiones relacionadas con el trabajo nocturno, en fines de semana, turnos, etc. Hemos observado que tanto el trabajo a turnos como las noches tienen consecuencias negativas en la salud y el bienestar de los trabajadores.

- La estructura del tiempo de trabajo: se refiere a todos aquellos aspectos sobre los cuales la compañía tenga posibilidad de alterar por ejemplo horarios, turnos, etc.

- Flexibilidad: contiene cuestiones relacionadas tanto con la conciliación laboral y familiar, así como el tener que emplear el tiempo de ocio para cubrir determinadas demandas de trabajo. 
En los resultados publicados por la EWCS (2015) este índice presenta diferencias por género mínimas. No obstante, del análisis del mismo se observa que el tiempo de trabajo semanal es reducido y esto se debe fundamentalmente al notable incremento del trabajo a tiempo parcial en los últimos años, lo cual implica menores ganancias y aumenta la inseguridad laboral de los trabajadores. Este incremento de la contratación a tiempo parcial ha sido tanto para hombres como para mujeres, ya que aunque la crisis económica ha afectado en mayor medida a los hombres en sectores como por ejemplo la construcción (sectores más masculinizados), las mujeres también se han visto afectadas por el hecho de tener menores ingresos en las familias debido a la crisis económica y tener que conciliar su vida familiar y laboral, hecho que sigue recayendo en mayor medida sobre la población femenina.

\subsection{Entorno social}

El entorno social es fundamental, en el ámbito del trabajo, para el crecimiento y desarrollo personal de los trabajadores. Este índice mide tanto aspectos positivos relacionados con el trabajo como son la calidad de la dirección en las organizaciones y el soporte social que reciben los trabajadores por parte de los compañeros y jefes a la hora de desarrollar su trabajo; como aspectos negativos del comportamiento social adverso que se pueda estar produciendo en las organizaciones, tales como abusos, humillaciones, violencia, etc. Dentro de este índice también se miden los aspectos de discriminación por sexo, edad, tipo de contrato, etc.

Analizando los resultados obtenidos en la EWCS (2015) podemos observar que apenas hay diferencias por género, aunque en términos de discriminación esta sigue siendo más acusada para las mujeres que para los hombres y además, observamos una mayor discriminación por edad. Con respecto al soporte de los compañeros y jefes, este índice presenta valores elevados, mientras que los comportamientos sociales adversos presentan niveles bastante bajos.

\subsection{Habilidades y discreción}

Este componente mide las habilidades requeridas en el trabajo para desempeñarlo, así como las oportunidades que pueden tener los trabajadores para influir en su trabajo o desarrollar diferentes destrezas a través de programas de formación. Este índice se mide a través de los siguientes factores:

- Dimensión cognitiva, es decir, la capacidad para aprender cosas nuevas, aplicar ideas propias, resolver problemas inesperados, tener autonomía para realizar tareas completas por sí mismo, etc. Todos estos aspectos tienen una influencia positiva en la motivación de los trabajadores. 
- Latitud de decisión, es decir, la habilidad para elegir o cambiar el orden de desarrollo de las tareas, la velocidad de las mismas o los métodos de trabajo, entre otros aspectos.

- La participación organizacional, es decir, tener la posibilidad de introducir mejoras, influir en las decisiones, etc.

- Las posibilidades de formación dentro de las diferentes organizaciones.

En términos globales, este índice ha mejorado en relación a los datos ofrecidos por la EWCS (2005), reduciéndose la brecha existente por género, ya que antes las mujeres no tenían tantas posibilidades de desarrollo profesional dentro de las organizaciones.

\subsection{Perspectivas de los trabajadores dentro de la organización}

Dentro de este índice se engloba tanto el estatus laboral, que tienen los trabajadores en función del tipo de contrato, el cual sabemos que tiene una repercusión directa sobre la seguridad que tienen los trabajadores dentro de las organizaciones, así como las perspectivas de carrera o desarrollo personal, la seguridad laboral que se mide no solo a través del estatus sino de aspectos más subjetivos como son el miedo a perder el empleo, y por último la cantidad de recortes o incrementos de plantilla que se han ido produciendo en las organizaciones en un periodo corto de tiempo.

Con respecto a estos factores utilizados para medir este índice, se observa que, aunque la brecha por género se ha ido reduciendo, los hombres continúan teniendo mayores posibilidades de desarrollo profesional que las mujeres.

\subsection{Ganancias recibidas}

Las ganancias recibidas, como ya se ha comentado anteriormente, es un índice que está calculado en euros y no está en una escala de 0 a 100, ya que mide las ganancias que obtienen los trabajadores tanto por cuenta ajena como los autónomos por el desarrollo de su trabajo. Mientras que el resto de componentes tiene un carácter multidimensional este goza de un carácter unidimensional. Este índice es un elemento clave dentro de la calidad de empleo, ya que la remuneración obtenida por el desempeño de trabajo es fundamental para los trabajadores. Por lo tanto, una remuneración adecuada implicará una mayor calidad de empleo, y por el contrario una remuneración baja, es decir, cuando un trabajador siente que se no se le paga acorde a su esfuerzo implicará una baja calidad de empleo, que repercutirá asimismo en la salud y el bienestar de los trabajadores.

Se observa que la distribución de las ganancias está sesgada ya que hay muchos trabajadores que presentan bajos niveles de renta. Además, existen grandes diferencias entre hombres y mujeres, lo cual se explica en gran medida 
porque las mujeres tienden a tener un mayor número de trabajos a tiempo parcial donde el número de horas de trabajo es más reducido (ILO, 2016).

\section{CONSTRUCCIÓN DEL INDICADOR SINTÉTICO}

Como ya se ha comentado anteriormente, nuestro objetivo es estimar un indicador sintético del concepto de calidad de empleo, mediante la agregación de las siete componentes recogidas por la EWCS del año 2015 en el informe publicado por Eurofound (2017). Existen una gran variedad de procedimientos y metodologías para el desarrollo de indicadores sintéticos como son las medidas de distancia, el análisis envolvente de datos y los métodos basados en las técnicas de análisis factorial, entre otras (Somarriba y Pena, 2009). No obstante, de entre todas las posibles opciones se ha seleccionado el índice $\mathrm{DP}_{2}$, pues verifica una serie de propiedades muy interesantes para el diseño de indicadores sintéticos. Esta metodología ha sido utilizada en diversos trabajos con un enfoque temporal y/o espacial (Cuenca et.al., 2010; Martín et.al., 2012; Martín et.al., 2013; Ray, 2014; Somarriba et.al., 2015; Somarriba y Zarzosa, 2016; Zarzosa y Somarriba, 2013, entre otros). Recientemente se ha aplicado la metodología con el fin de diseñar indicadores a nivel individual como en Somarriba y Zarzosa (2018) y Pinillos-Franco y Somarriba (2018). En dichos trabajos, emplean la medida a nivel individual, lo que permite enriquecer el análisis, ya que nos da la posibilidad de realizar comparaciones entre subgrupos de población.

$\mathrm{La} \mathrm{DP}_{2}$ se define de la siguiente manera:

$$
D P_{2}=\sum_{i=1}^{n}\left\{(d i / \sigma i)\left(1-R_{i, i-1, \ldots, 1}^{2}\right)\right\} \operatorname{con} \mathrm{R}_{1}^{2}=0
$$

Sea $X=\left\{X_{i j}\right\}$ la matriz de datos correspondientes a las $\mathrm{m}$ individuos (filas) $\mathrm{y}$ los $\mathrm{n}$ indicadores (columnas) y sea $\mathrm{X}_{\mathrm{ij}}$ el estado en que se encuentra el componente i-ésimo en el individuo j.

$i=1,2, \ldots, \mathrm{n}$

$j=1,2, \ldots \mathrm{m}$

Notemos por $\mathrm{X} *=[\mathrm{X} * 1, \mathrm{X} * 2, \ldots, \mathrm{X} * \mathrm{~m}]$ el estado de la base de referencia respecto a los $\mathrm{n}$ componentes. Se toma como base de referencia un individuo teórico que alcanza los peores valores de los indicadores objeto de estudio. Por tanto, el indicador $\mathrm{DP}_{2}$ devuelve las distancias de cada individuo respecto a ese individuo teórico de referencia; por ello un valor más elevado de la medida de distancia indica que el individuo está más alejado de ese valor mínimo de calidad del trabajo. 
Se considera

$$
d_{i}=\left|X_{i j}-X_{i *}\right|
$$

y sean:

- $\quad m$ el número de individuos.

- $n$ el número de variables.

- $\mathrm{X}_{\mathrm{ij}}$ el valor de la variable i en el individuo j.

- $\sigma_{\mathrm{i}}$ la desviación típica de la variable $\mathrm{i}$.

- $\mathrm{R}_{\mathrm{i}, \mathrm{i}-1, \ldots, 1}^{2}$ el coeficiente de determinación en la Regresión de $\mathrm{X}_{\mathrm{i}}$ sobre $\mathrm{X}_{\mathrm{i}-1,1}$, $\mathrm{X}_{\mathrm{i}-2}, \mathrm{X}_{1}$.

- (1- $\left.\mathrm{R}_{\mathrm{i}, \mathrm{i}-1, \ldots, 1}^{2}\right)$ actúa a modo de factor corrector que elimina la información duplicada.

La $\mathrm{DP}_{2}$ cumple todas las propiedades exigibles a un buen indicador de distancia y permite, además, comparaciones intertemporales, interespaciales e interindividuales.

A raíz del cumplimiento de una serie de propiedades del indicador sintético, el indicador de intensidad del trabajo cuyo aumento implica un empeoramiento de la calidad de empleo, se multiplica por -1 de forma que un aumento del valor de cualquier indicador suponga una mejora de la calidad de empleo y en ningún caso un empeoramiento de la misma.

Además, obtenemos el Coeficiente de Discriminación (CD) para poder determinar el poder de discriminación que tiene cada indicador parcial sobre los resultados globales de la calidad de empleo, y poder determinar si la información proporcionada por cada indicador está más o menos dispersa entre los diferentes individuos de los distintos países de la Unión Europea. Este coeficiente de determinación según Ivanovic en 1974 se determina de la siguiente manera:

$$
C D_{i}=\frac{2}{m(m-1)} \sum_{j, l>j}^{k i} m_{i j} m_{i l}\left|\frac{X_{i j}-X_{i l}}{\overline{X_{l}}}\right| \text {, }
$$

donde m es el número de individuos.

Los valores de este coeficiente estudiado en profundidad por Zarzosa (1994, 1996) y por Somarriba y Zarzosa (2016), oscilan entre 0 y 2 . El 0 nos indica que el indicador parcial estudiado no tiene ningún poder discriminatorio, es decir, toma el mismo valor para todos los individuos, mientras un coeficiente de discriminación de 2 implica la máxima potencia discriminante. En el siguiente apartado, se analizará el poder discriminatorio de cada uno de los índices que van a componer nuestro indicador sintético. 
Nuestro estudio se realiza a partir de la EWCS (2015), que es una encuesta que se desarrolla a nivel de la UE cada 5 años desde 1990 con el fin de proporcionar una panorámica general sobre las condiciones de trabajo en Europa y poder a su vez identificar y cuantificar las condiciones de trabajo, determinar los posibles grupos de riesgo, contribuir a la formulación de políticas europeas, etc. Para nuestro trabajo hemos utilizado una muestra de 12.978 hombres $(48,1 \%)$ y 13.984 mujeres $(51,9 \%)$.

\section{ESTRUCTURA DEL INDICADOR SINTÉTICO DE CALIDAD DEL EMPLEO (ISCE)}

La siguiente tabla recoge la estructura del indicador sintético, figurando en la primera columna el valor del coeficiente de correlación en valor absoluto entre los diferentes indicadores y nuestro ISCE, valor que marca el orden de acceso de los diferentes indicadores a la estructura del indicador. En la segunda columna, aparece el factor corrector que nos muestra el porcentaje de información nueva que cada indicador incorpora al indicador sintético descontando el efecto de los indicadores previamente introducidos. Y por último, en la tercera columna aparece el Coeficiente de Discriminación, que nos indica el poder discriminatorio que tiene cada uno de los indicadores dentro del ISCE y que nos va a permitir observar si existen grandes diferencias entre los individuos de los diferentes países de la UE28.

Tabla 1

Estructura del ISCE

\begin{tabular}{|l|c|c|c|}
\hline \multicolumn{1}{|c|}{ VARIABLES } & $|r|$ & $\mathbf{( 1 - R}^{2} \mathbf{)}$ & CD \\
\hline Entorno físico & 0,6051 & 1 & 0,1857 \\
\hline Perspectivas de los trabajadores & 0,5716 & 0,9823 & 0,3454 \\
\hline Entorno social & 0,5449 & 0,9572 & 0,3226 \\
\hline Habilidades y discreción en el trabajo & 0,5387 & 0,8496 & 0,4471 \\
\hline Intensidad del trabajo & 0,4682 & 0,8163 & 0,6310 \\
\hline Calidad del tiempo de trabajo & 0,4454 & 0,8729 & 0,2130 \\
\hline Ganancias recibidas & 0,3921 & 0,8075 & 0,6619 \\
\hline
\end{tabular}

Fuente: Elaboración propia.

En la estructura del indicador sintético se observa que todos los indicadores parciales incorporan una gran cantidad de información al indicador, ya que los factores correctores oscilan entre 0,8075 y 1 , por lo que todas las variables son significativas para la construcción del mismo, ello nos demuestra que todas las dimensiones resultan esenciales en la medición de la calidad del empleo. La variable que incorpora el $100 \%$ de la información es el entorno físico, que guarda relación con los riesgos ergonómicos derivados de las posturas y movimientos, 
los riesgos derivados del ambiente y los riesgos químicos y biológicos, siendo los primeros los más frecuentes en los trabajadores de la UE. Este a su vez, es el índice que presenta una correlación más fuerte con la calidad del empleo. En el lado opuesto, tenemos la variable ganancias recibidas que es la que presenta una correlación más débil, aunque aporta una gran cantidad de información al indicador sintético (81\%).

Por el contrario, si nos fijamos en los CD obtenidos, los cuales figuran en la última columna de la tabla, se observa que tanto el índice de ganancias recibidas, como el índice de intensidad en el trabajo son los que tienen un poder discriminatorio más fuerte, es decir, en estos términos se observan mayores disparidades entre los diferentes individuos de la UE28. Sin embargo, el índice del entorno físico, que es uno de los que presenta una correlación más fuerte con el indicador sintético, es el que tiene un poder de discriminación más débil. Asimismo, otro de los índices con un CD bastante bajo es la calidad del tiempo de trabajo, que a diferencia de lo que se pueda pensar no presenta grandes desigualdades.

\section{DISTRIBUCIÓN DEL ISCE Y SUS COMPONENTES. PRINCIPALES ESTADÍSTICOS}

Si se analizan los principales estadísticos de las diferentes componentes y del propio indicador sintético (ISCE), se observa que como ya se había comentado anteriormente, todos los índices con la excepción del de ganancias oscilan entre 0 y 100, siendo 0 la puntuación más desfavorable y 100 la más favorable. En términos medios, destacar la intensidad del trabajo ya que es la componente que presenta la puntuación más baja de 33,83 puntos, tal y como se observa en la Tabla 2. Por el contrario, el entorno físico junto con el entorno social y la calidad del tiempo de trabajo son los índices que presentan una mayor puntuación y por tanto son los aspectos mejor valorados por los trabajadores. No obstante, el coeficiente de variación (CV) nos muestra que la media es poco significativa en todas las componentes, con la excepción de la componente ganancias.

En términos de asimetría, prácticamente todas las variables son simétricas porque sus coeficientes de asimetría presentan valores muy próximos a 0 con la excepción del índice de ganancias, el cual presenta una asimetría positiva muy acusada lo que nos indica la presencia de rentas anormalmente altas. Asimismo, este indicador presenta un alto grado de curtosis con una alta concentración de valores.

En conclusión, las componentes con mejores puntuaciones son el entorno social y físico, junto con la calidad del tiempo de trabajo. Estas como ya habíamos visto anteriormente, eran variables fuertemente relacionadas con el indicador sintético. Por el contrario, la intensidad del tiempo de trabajo es la componente con peor resultado. A su vez, hemos observado que el ISCE no 
presenta valores muy elevados en términos de media y mediana por lo que los niveles de calidad de empleo en la UE28 no son muy elevados en términos generales.

Tabla 2

Principales estadísticos

\begin{tabular}{|c|c|c|c|c|c|c|c|c|}
\hline & Ganancias & $\begin{array}{c}\text { Habilidades } \\
\text { y } \\
\text { discreción }\end{array}$ & $\begin{array}{c}\text { Entorno } \\
\text { social }\end{array}$ & $\begin{array}{l}\text { Entorno } \\
\text { físico }\end{array}$ & $\begin{array}{l}\text { Intensidad } \\
\text { del Trabajo }\end{array}$ & Perspectivas & $\begin{array}{l}\text { Calidad del } \\
\text { tiempo de } \\
\text { trabajo }\end{array}$ & ISCE \\
\hline Mínimo & 0,48 & 0,00 & 0,00 & 0,00 & 0,00 & 0,00 & 6,41 & 10,83 \\
\hline Máximo & $32.618,82$ & 100,00 & 100,00 & 100,00 & 100,00 & 100,00 & 100,00 & 50,86 \\
\hline Media & $1.379,09$ & 55,27 & 77,16 & 83,20 & 33,83 & 63,36 & 70,62 & 35,29 \\
\hline Mediana & $1.211,56$ & 57,50 & 86,11 & 87,18 & 31,67 & 62,50 & 74,29 & 35,02 \\
\hline $\begin{array}{l}\text { Desviación } \\
\text { estándar }\end{array}$ & 958,62 & 21,64 & 24,03 & 14,56 & 18,88 & 19,49 & 13,89 & 3,23 \\
\hline Varianza & $918.944,49$ & 468,45 & 577,39 & 212,03 & 356,47 & 379,99 & 192,86 & 10,46 \\
\hline $\begin{array}{l}\begin{array}{l}\text { Coeficiente } \\
\text { de Variación }\end{array} \\
\end{array}$ & 0,70 & 0,39 & 0,31 & 0,18 & 0,56 & 0,31 & 0,20 & 0,09 \\
\hline Asimetría & 5,15 & $-0,34$ & $-1,29$ & $-1,34$ & 0,50 & $-0,42$ & $-1,08$ & 0,39 \\
\hline $\begin{array}{l}\text { Error } \\
\text { estándar de } \\
\text { asimetría }\end{array}$ & 0,01 & 0,01 & 0,01 & 0,01 & 0,01 & 0,01 & 0,01 & 0,01 \\
\hline Curtosis & 96,12 & $-0,69$ & 0,61 & 2,03 & $-0,25$ & $-0,04$ & 0,83 & 0,67 \\
\hline $\begin{array}{l}\text { Error } \\
\text { estándar de } \\
\text { curtosis }\end{array}$ & 0,03 & 0,03 & 0,03 & 0,03 & 0,03 & 0,03 & 0,03 & 0,03 \\
\hline
\end{tabular}

Fuente: Elaboración propia, EWCS.

Si se analizan las puntuaciones por género de los diferentes indicadores, para el conjunto de países de la UE28 (Tabla 3), se observa que las puntuaciones medias de las diferentes variables son muy parecidas aunque cabe destacar que en los indicadores del entorno físico y la calidad del tiempo de trabajo los hombres presentan valores más elevados, por lo que presentan mejores condiciones en estos aspectos que las mujeres. Destacar sobre todo la gran diferencia que existe por género cuando nos referimos a la variable ganancias recibidas, es decir, al salario o retribución percibida por el desempeño de su trabajo. En este caso, los hombres ganan de media 1.587,78€ mientras que las mujeres únicamente ganan $1.185,47 €$. Esto se explica por la brecha salarial existente entre hombres y mujeres. Según datos de la Comisión Europea (CE) publicados en octubre de 2017, "el salario bruto de las mujeres en la UE es un 16,3\% inferior al de los hombres". La CE destaca las siguientes causas como determinantes de la existencia de la brecha salarial entre hombres y mujeres: los puestos de gestión y control siguen estando ocupados mayoritariamente por hombres; las mujeres tienen una mayor carga de trabajo no remunerado que los hombres; la segregación existente en el mercado laboral y la enseñanza; y la discriminación salarial que sigue estando presente en una gran cantidad de países. 
Tabla 3

Media por sexo de las variables del ISCE en la UE

\begin{tabular}{|l|c|c|}
\hline \multicolumn{1}{|c|}{ Variables del ISCE } & Media hombres & Media mujeres \\
\hline Ganancias recibidas & $1.587,78$ & $1.185,47$ \\
\hline Habilidades y discreción en el trabajo & 55,50 & 55,05 \\
\hline Entorno social & 77,99 & 76,39 \\
\hline Entorno físico & 80,35 & 85,84 \\
\hline Intensidad del trabajo & 34,01 & 33,67 \\
\hline Perspectivas de los trabajadores & 63,81 & 62,94 \\
\hline Calidad del tiempo de trabajo & 68,85 & 72,26 \\
\hline
\end{tabular}

Fuente: Elaboración propia, EWCS.

Por último, si observamos el histograma de frecuencias del ISCE por género (Figura 2) podemos ver que para los hombres el indicador sintético es bastante simétrico y se encuentra más centrado en torno a la media de 35,37 puntos, y sin embargo en el caso de las mujeres presenta una asimetría positiva, lo que implica que hay un mayor número de mujeres situadas por encima de la media del indicador sintético.

Figura 2

Histograma por género del ISCE

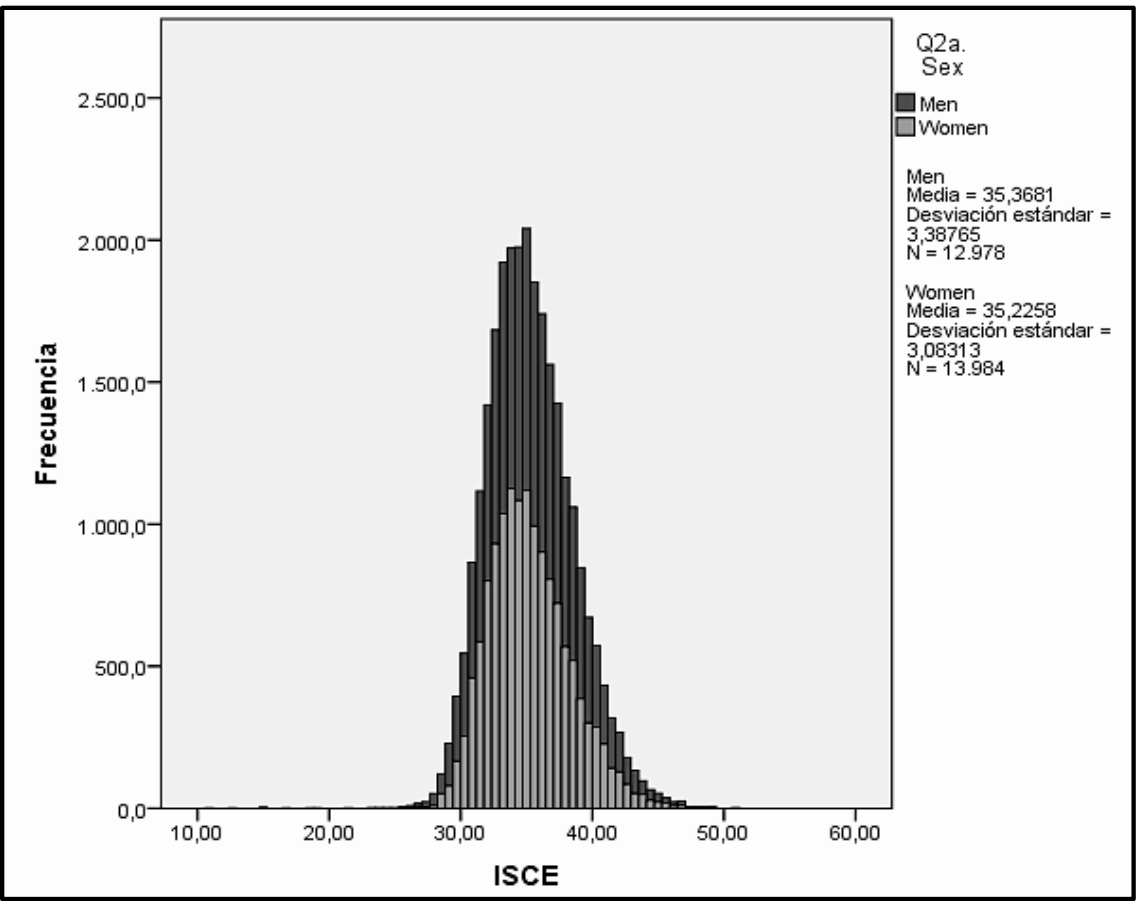

Fuente: Elaboración propia, SPSS. 


\section{DISTRIBUCIÓN POR PAÍSES Y GÉNERO DEL INDICADOR SINTÉTICO DE CALIDAD DE EMPLEO}

En la siguiente tabla se recoge el valor medio por género y país del ISCE y si dicha diferencia es o no significativa (Prueba T-Student). La diferencia calculada se obtiene comparando el ISCE de las mujeres menos el ISCE de los hombres.

Se observa que la diferencia por género es significativa en 15 de los 28 países de la Unión Europea que componen la EWCS (2015), es decir, se observan diferencias entre la calidad de empleo entre hombres y mujeres en la mitad de los países. Asimismo, en términos globales dicha diferencia también es significativa por lo que se puede afirmar que los hombres presentan mejores índices de calidad de empleo que las mujeres en la UE28.

Del análisis de los resultados obtenidos observamos que las diferencias en calidad de empleo entre hombres y mujeres en la UE28 pueden estar determinadas por los diferentes estados de bienestar existentes en Europa, ya que las políticas que se llevan a cabo en materia de empleo repercuten a su vez sobre la salud y el bienestar de los trabajadores (Moore et.al., 2006; Bambra, 2007; Bambra et.al., 2009; Heinz y Lund, 2012 y Holman, 2013). Diversos estudios han seguido la primera clasificación que hizo Esping-Andersen (1990) de los países de la OCDE, el cual señalaba los siguientes grupos de países:

- Democrático: en este se incluyen los países nórdicos como Dinamarca, Finlandia, Países Bajos y Suecia.

- Continental: dentro de este tenemos los países más conservadores como Alemania, Austria, Francia, Luxemburgo y Bélgica.

- Liberal: integrado por el Reino Unido e Irlanda.

- Del sur de Europa: donde tenemos a España, Grecia, Italia, Portugal, Chipre, Malta y Croacia.

- De Europa del Este y países de reciente incorporación a la UE28: estos son algunos como República Checa, Eslovaquia, Eslovenia, Polonia, Letonia, Bulgaria, Lituania, Rumanía, Estonia y Hungría. Este régimen no estaba recogido dentro de la clasificación de Esping-Andersen (1990) ni de los estudios comentados anteriormente porque estos países son de reciente incorporación en la UE.

A la vista de los resultados del ISCE (Tabla 4) es en los países con régimen democrático y continental donde apreciamos que las mujeres tienen un ISCE superior a los hombres, con la excepción de Austria. En 5 de los 7 países donde se cumple esta premisa las diferencias de género resultan significativas (Prueba T-student). En el lado opuesto, encontramos los países de la zona este y de reciente incorporación a la UE, en los cuales el gap es significativo en 6 de los 10 países, siendo el ISCE de los hombres superior al de las mujeres, por lo que 
en estos regímenes los hombres presentan una mejor calidad de empleo que las mujeres. Por otra parte, en los países del régimen liberal, Reino Unido e Irlanda, el gap de género no es significativo, por lo que no existen diferencias en materia de calidad de empleo. Por último, en los países del sur de Europa, la diferencia por género únicamente es significativa en el caso de España, Croacia, Chipre y Malta donde se observa que los hombres presentan una calidad de empleo superior a la de las mujeres. En términos globales de la UE28 podemos concluir que los hombres presentan índices de calidad de empleo superiores al de las mujeres.

Tabla 4

Indicador Sintético de Calidad de Empleo por países y género

\begin{tabular}{|c|c|c|c|c|}
\hline País & ISCE $_{M}$ & ISCE $_{H}$ & Diferencia & p-valor \\
\hline Dinamarca & 34,2262 & 33,3796 & 0,8466 & 0,000 \\
\hline Países Bajos & 35,0497 & 34,3672 & 0,6825 & 0,002 \\
\hline Luxemburgo & 34,326 & 33,7164 & 0,6096 & 0,020 \\
\hline Finlandia & 34,7363 & 34,1944 & 0,5419 & 0,008 \\
\hline Suecia & 34,7893 & 34,2908 & 0,4985 & 0,014 \\
\hline Francia & 35,6086 & 35,358 & 0,2506 & 0,182 \\
\hline Alemania & 35,1153 & 34,943 & 0,1723 & 0,243 \\
\hline Bélgica & 34,4944 & 34,4996 & $-0,0052$ & 0,966 \\
\hline Reino Unido & 34,6556 & 34,7032 & $-0,0476$ & 0,796 \\
\hline Eslovaquia & 35,7572 & 35,8643 & $-0,1071$ & 0,650 \\
\hline Austria & 34,8569 & 34,9692 & $-0,1123$ & 0,633 \\
\hline Irlanda & 34,4402 & 34,5923 & $-0,1521$ & 0,508 \\
\hline República Checa & 35,5165 & 35,7098 & $-0,1933$ & 0,408 \\
\hline Portugal & 35,4495 & 35,7026 & $-0,2531$ & 0,254 \\
\hline Eslovenia & 35,3356 & 35,591 & $-0,2554$ & 0,166 \\
\hline Grecia & 37,3438 & 37,6163 & $-0,2725$ & 0,297 \\
\hline Letonia & 35,674 & 36,0066 & $-0,3326$ & 0,130 \\
\hline Italia & 35,6407 & 36,0166 & $-0,3759$ & 0,073 \\
\hline Malta & 34,3343 & 34,7413 & $-0,407$ & 0,046 \\
\hline España & 36,0793 & 36,5156 & $-0,4363$ & 0,002 \\
\hline Lituania & 35,0787 & 35,55 & $-0,4713$ & 0,032 \\
\hline Croacia & 35,9605 & 36,4835 & $-0,523$ & 0,041 \\
\hline Chipre & 36,1734 & 36,7192 & $-0,5458$ & 0,009 \\
\hline Rumanía & 36,2987 & 36,8726 & $-0,5739$ & 0,005 \\
\hline Estonia & 34,7677 & 35,5612 & $-0,7935$ & 0,000 \\
\hline Bulgaria & 34,8914 & 35,8146 & $-0,9232$ & 0,000 \\
\hline Hungría & 35,4332 & 36,5785 & $-1,1453$ & 0,000 \\
\hline Polonia & 35,3136 & 36,7141 & $-1,4005$ & 0,000 \\
\hline EU 28 & 35,2557 & 35,3775 & $-0,1218$ & 0,000 \\
\hline
\end{tabular}

${ }^{*} p<0,05,{ }^{\star \star} p<0,01,{ }^{\star \star \star} p<0,001$

Fuente: Elaboración propia.

A continuación, en la Figura 3 se han representado las diferencias entre el 
ISCE de las mujeres y el de los hombres. Las zonas más oscuras son aquellas donde las mujeres tienen una calidad de empleo superior a la de los hombres y por el contrario, las zonas más claras son aquellas donde los hombres presentan mejores índices de calidad de empleo. Como ya hemos comentado en el análisis de los datos, las mujeres presentan niveles de calidad de empleo superior en los países nórdicos y de Europa Central. No obstante, en los países del sur y de la zona este son los hombres los que tienen una calidad de empleo superior.

Figura 3

Diferencias en el ISCE por género en Europa

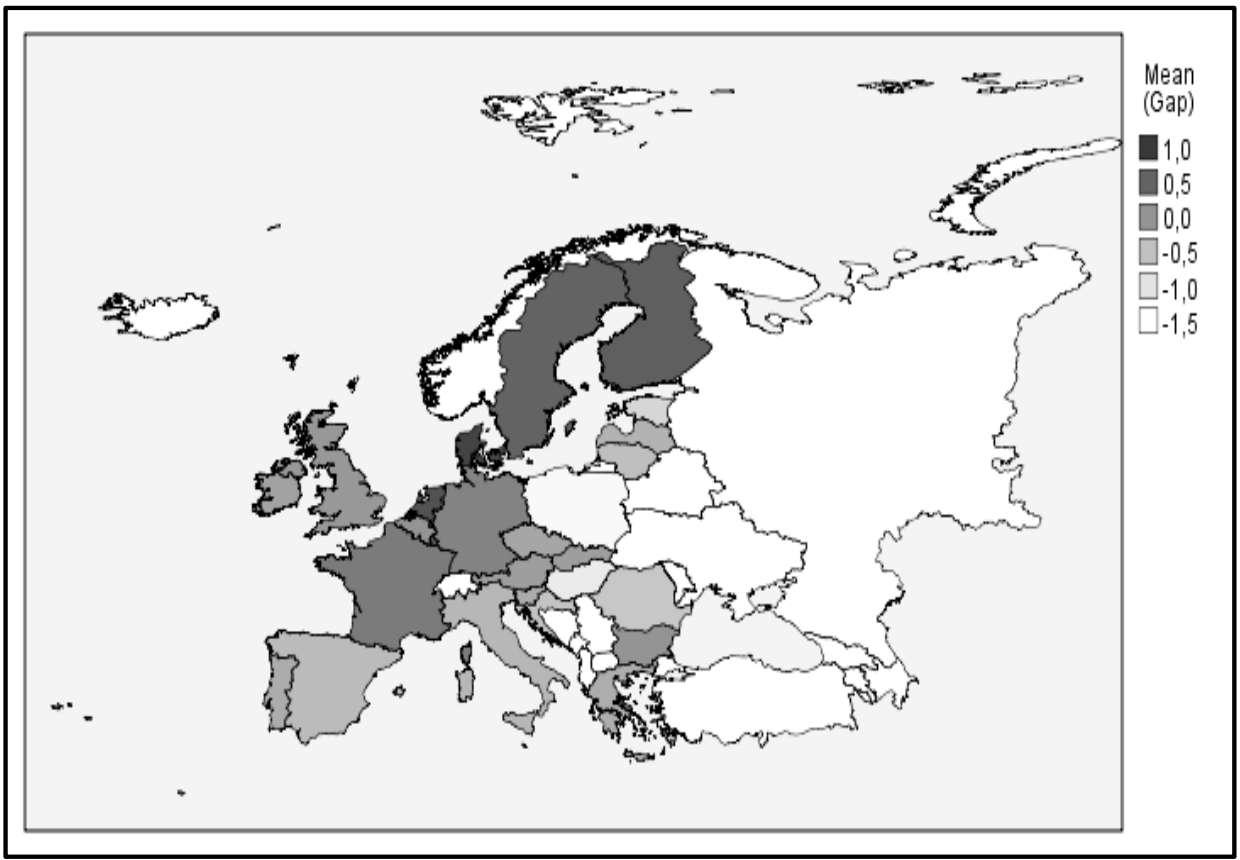

Fuente: Elaboración propia.

\section{CONCLUSIONES}

Este trabajo nos ha permitido construir un nuevo Indicador Sintético de Calidad de Empleo (ISCE) mediante la agregación de las diferentes componentes que contemplaba la EWCS (2015), en el informe publicado por Eurofound (2017) como indicadores de los niveles de calidad de empleo de los individuos de la UE28. Este nuevo ISCE lo hemos estimado a través de la metodología $\mathrm{DP}_{2}$ ya que la misma presenta una serie de características idóneas para nuestro estudio, como que nos permite hacer no solo análisis intertespaciales sino también interindividuales. Gracias a esta metodología hemos construido un ISCE mediante la agregación de siete componentes, y hemos podido detectar las 
diferencias existentes por género en la calidad de empleo entre los diferentes países de la UE. Hemos observado, que en la mitad de los países de la UE hay diferencias significativas para explicar las desigualdades existentes por género, siendo la calidad de empleo de las mujeres superior a la de los hombres en los países nórdicos y de Europa Central, y por el contrario estas presentan índices inferiores a los hombres en los países de la zona sur y del este de Europa. Esto se debe en gran medida a los regímenes de bienestar existentes en cada uno de los territorios de Europa, que hace que se desarrollen diferentes tipos de políticas en materia de empleo, bienestar, salud, etc. Estos regímenes los determinó EspingAndersen (1990) y los han aplicado también en sus estudios Moore et.al. (2006), Bambra (2007), Bambra et.al. (2009) y Heinz y Lund (2012).

Por otra parte, se ha observado que aunque todas las componentes son relevantes en la estimación del ISCE puesto que todas aportan una gran cantidad de información y tienen un elevado grado de correlación con el ISCE, el entorno físico ha sido la más importante. Asimismo, del análisis de los principales estadísticos hemos corroborado que esta variable junto con el entorno social y la calidad del tiempo de trabajo son las que obtienen mejores puntuaciones. Mientras que la intensidad del trabajo es uno de los índices con peores resultados. Esto se ha producido por la necesidad de las organizaciones de adaptarse a las fluctuaciones de los mercados y a las tendencias económicas en general, que se han producido en los últimos años incrementándose la intensidad del trabajo (Anttila et.al., 2015). Por lo tanto, los elevados niveles de intensidad no solo afectan a la calidad del empleo, sino que tienen una repercusión directa sobre la calidad de vida y especialmente sobre la salud de los trabajadores (Zajc y Kohont, 2017). Según establecían estos autores, los elevados niveles de intensidad se llevan a cabo con el objetivo de obtener mayores ganancias, pero el hecho de tener que trabajar más horas para conseguirlo hace que afecte negativamente a la salud de los trabajadores incrementando el estrés, entre otros factores.

Asimismo, hemos podido apreciar que para la mayoría de las componentes que conforman nuestro indicador sintético los hombres presentan mejores puntuaciones que las mujeres, lo cual tiene una relación directa con el hecho de que los hombres presenten niveles de calidad de empleo más elevados que las mujeres en los países de la UE28. Esto lo corrobra el estudio llevado a cabo por Crespo et.al. (2017). Por el contrario, en los índices del entorno físico y la calidad del tiempo de trabajo son las mujeres las que presentan mejores puntuaciones. Destacar sobre todo la gran diferencia existente por género cuando nos centramos en la variable ganancias recibidas, ya que aunque se están impulsando políticas para evitar estas desigualdades, seguimos observando que las mujeres tienen salarios medios bastante inferiores a los de los hombres.

En conclusión, a pesar de las políticas que se están desarrollando para lograr una igualdad en materia de empleo entre hombres y mujeres, seguimos 
detectando diferencias significativas entre ambos grupos. Por lo tanto, las agendas sociales de los gobiernos nacionales de los diferentes países europeos deben seguir luchando para conseguir no solo una igualdad entre hombres y mujeres sino mejorar la calidad del empleo, puesto que esto tiene un impacto positivo no solo sobre el trabajo y por consiguiente sobre los beneficios empresariales y la economía, sino sobre la calidad de vida y la salud de los trabajadores.

\section{REFERENCIAS BIBLIOGRÁFICAS}

ANTTILA, T.; OINAS, T.; TAMMELIN, M.; NÄTTI, J. (2015). "Working-Time Regimes and Work-Life Balance in Europe". European Sociological Review 31(6), pp. 713-724

ARRANZ, J.M., GARCÍA-SERRANO, C. Y HERNANZ, V. (2016). Índice de calidad de empleo. Asempleo, Madrid.

BAMBRA, C. (2007). "Sifting the wheat from the chaff: a two-dimensional discriminant analysis of welfare state regime theory". Soc Policy Admin, 41, pp. 1-2

BAMBRA, C.; POPE, D.; SWAMI, V.; et al. (2009). "Gender, health inequalities and welfare state regimes: a cross-national study of 13 European countries". Journal of Epidemiology \& Community Health 63, pp.38-44.

BEGEGA, S.G.; RODRIGUEZ, A.M.G. (2009). "La calidad del empleo en la Unión Europea. Debate político y construcción de indicadores". Revista del ministerio de Trabajo e Inmigración, 81, pp, 71-88.

CLARK, A. (2005). "Measures of job satisfaction: what makes a good job? Evidence from OECD Countries". OECD Labour Market and Social Policy Occasional Papers, $n \div 34$, Paris (OCDE Directorate for Employment, Labour and Social Affairs).

COMISIÓN EUROPEA (2017). La brecha salarial entre géneros en España.

CRESPO, N.; SIMOES, N.; PINTO, J. C. (2017). "Determinant factors of job quality in Europe". Argumenta Oeconomica, 1, pp. 15-40.

CUENCA GARCÍA, E.; RODRIGUEZ MARTÍN, J.A.; NAVARRO PABSDORF, M. (2010). "The Features of Development in the Pacific Countries of the African, Caribbean and Pacific Group". Social Indicators Research, 99(3), pp. 469-485.

ESPING-ANDERSEN G. (1990). "The Three Political Economies of the Welfare State". International journal of sociology, 20(3), pp. 92-123.

EUROFOUND (2015). "Encuesta Europea sobre las condiciones de trabajo". Disponible en: https://www.eurofound.europa.eu/es/surveys/european-working-conditions-surveys

EUROFOUND (2017). "Sixth European Working Conditions Survey - Overview report (2017 update)". Publications Office of the European Union, Luxembourg.

EUROPEAN COMMISSION (2017). "2017 Report on Equality between Women and Men in the European Union".

EUROPEAN TRADE UNION INSTITUTE (ETUI). "Job Quality Index (JQI), Labour Market, Employment and Social Policy". Disponible en: https://www.etui.org/Topics/Labourmarket-employment-social-policy/Job-quality-index-JQI

GREEN, F. (2006). "Demanding work. The paradox of job quality in the affluent economy". 
Princeton University Press, pp. 14-15.

HEINZ, J.; LUND, F. (2012). "Regímenes del bienestar y política social: revisando el papel del trabajo y el empleo". Avances de investigación, 81, Fundación Carolina

HOLMAN, D. (2013). "Job types and job quality in Europe". Human Relations, 66(4), pp. 475-502.

ILO. (2016). "Women at Work, Trends 2016". International Labour Office-Geneva, Vol. 42.

IVANOVIC, B. (1974). "Comment établir une liste des indicateurs de développement". Reveu de Statistique Appliquée, XXII, 2.

MARTÍN, J. A. R.; FERNÁNDEZ, J. A. S. (2012). "An index of maternal and child health in the least developed countries of Asia". Gaceta Sanitaria, 26(2), pp. 190-192.

MARTíN, J.A.R.; MOLINA, M.D.M.H.; FERNÁNDEZ, J.A.S.(2013). "An Index of Progress Towards the MDG1 in Southern Africa and the Horn of Africa". Applied Research in Quality of Life, 8(4), pp. 467-480.

MOORE, K.; ET.AL. (2006). "Measuring the Quality of Employment in the EU". Ethnicity \& Disease 16(4), pp. 772-777

PIASNA, A. (2018). “'Bad jobs' recovery? European Job Quality Index 2005-2015”. ETUI Research Paper - Working Paper 2017.06.

PINILLOS-FRANCO, S.; SOMARRIBA-ARECHAVALA, N. (2018). "A proposal for a Synthetic Health Indicator in the European Union: an Analysis of Gender Health Inequalities". Applied Research in Quality of Life, pp. 1-15.

RAY, S. (2014). "An index of maternal and child healthcare status in India: Measuring interand intra-state variations from capability perspectives". Social indicators research, 117(1), pp. 195-207.

SOMAVIA, J. (2004). "A fair globalization". The role of the ILO, Report of the DirectorGeneral on the Commission on the social dimension of globalization, Geneva: International Labour Organization.

SOMARRIBA ARECHAVALA, N.; MERINO LLORENTE, M.C.; RAMOS TRUCHERO, G.; NEGRO MACHO, A. (2010). "La calidad del trabajo en la Unión Europea". Estudios de Economía Aplicada, 28(3) pp. 1-22.

SOMARRIBA, N.; PENA, B. (2009). "Synthetic indicators of quality of life in Europe". Social Indicators Research, 94(1), pp. 115-133.

SOMARRIBA, N.; ZARZOSA, P.; PENA, B. (2015). "The economic crisis and its effects on the quality of life in the European Union". Social Indicators Research, 120, pp. 323-343.

SOMARRIBA, N.; ZARZOSA, P. (2016). "Quality of life in Latin America: A proposal for a synthetic Indicator". Indicators of Quality of Life in Latin America. Social Indicators Research Serie, 62, pp. 19-56.

SOMARRIBA, N.; ZARZOSA, P. (2018). "Quality of Life in the European Union: an econometric analysis from a gender perspective". Social Indicators Research, pp. 1-22.

ZAJC, J. C.; KOHONT, A. (2017). "Impacts of Work Intensity on Employees' Quality of Work, Life and Health". Teorija in Praksa 54(2), pp. 209-223.

ZARZOSA, P. (1994): "El criterio de discriminación en la selección de indicadores de bienestar. Análisis del coeficiente de Discriminación de Ivanovic". Estudios de Economía Aplicada, 2, 169-185.

ZARZOSA, P. (1996). "Aproximación a la medición del bienestar social. Idoneidad del indicador sintético "Distancia-P2". Cuadernos de Economía, 24, pp. 139-163.

ZARZOSA, P; SOMARRIBA, N. (2013). "An assessment of social welfare in Spain: 
Territorial analysis using a synthetic welfare Indicator". Social Indicators Research, 111(1), pp. 1-23. 